\title{
CONCERNING THE COMPLEMENTARY INTERVALS OF COUNTABLE CLOSED SETS*
}

BY J. R. KLINE

Professor R. L. Moore† recently gave an example showing that the following statement due to Hobson $\$$ is incorrect:

"A non-dense closed set is enumerable if its complementary intervals are such that every one of them abuts on another one at each of its ends."

In considering this example, we notice that while each of the complementary intervals of the original set of Moore's example are such that each one of them abuts on another one at each of its ends, the same is not true if we consider the complementary intervals of $G^{\prime}$, the first derived set $\S$ of $G$. This leads us to the following theorem:

THeorem A. A non-dense closed set $G$ is enumerable, if $G$ and every derived set of $G$ has the property that every one of its complementary intervals is such that it abuts on another one at each of its ends.

Proof: Let us suppose that $G$ is not enumerable. Then there exists a number $\beta$ of the first or second class such that the derived set $G^{\beta+1}$ is identical with the derived set $G^{\beta}$ and $G^{\beta}$ is perfect. $\|$ It follows that $G^{\beta}$ has no

* Presented to the Society, October 25, 1924.

† See R. L. Moore, An uncountable, closed, and non-dense point set, each of whose complementary intervals abuts on another one at each of its ends, this Bulletis, vol. 29, (1923), pp. 49-50.

‡ See E. W. Hobson, The Theory of Functions of a Real Variable 1st edition (1907), p. 92; and $2 d$ edition (1921), p. 113.

$\S$ If $G$ is a point set, then the set $G^{\prime}$ consisting of all limit points of $G$ is called the derived set or the derivative of $G$.

II See E. W. Hobson, loc. cit., p. 115. In his proof of the theorem that every non-dense linear closed set is, in general, made up of an enumerable set and a perfect set, Hobson here shows that unless the non-dense linear closed set $G$ is enumerable, there is a number $\beta$ of the first or second class such that $G^{\beta}$ is perfect. 
isolated points. As $G$ is non-dense and closed, it follows that $G^{\beta}$ is non-dense. Select a complementary interval of $G^{\beta}$. As this interval abuts on another complementary interval of $G^{\beta}$ at both its ends, it follows that the supposition that $G$ is not enumerable has lead to a contradiction.

As Hobson points out, it is not necessary that each complementary interval abut on another at each of its ends in order that a set be enumerable. But as no subset of a closed countable set is perfect, ${ }^{*}$ then there must be some definite number $\beta$ of the first or second class such that $G^{\beta+1}$ vanishes. Thus $G^{\beta}$ consists only of isolated points. It follows that its complementary intervals abut at both their ends. Thus we are enabled to state the following theorem. Theorem B. In order that a linear closed set be enumerable it is both necessary and sufficient that there exist some number $\beta$ of the first or second class such that $G^{\beta}$ and all the derived sets of $G$ after $G^{\beta}$ have the property that each of its complementary intervals abuts on another one at both its ends.

University of Pennsyluania

* See R. L. Moore, An extension of the theorem that no countable point set is perfect, Proceedings of the National Academy, vol. 10, pp. 168-170. 